\title{
The Neuromuscular Efficiency of Trunk Muscles is Altered by Age in the Abdominal Muscles but by Gender in the Back Muscles
}

\author{
Christoph Anders ${ }^{1 *}$, Agnes Huebner ${ }^{1}$ and Bernd Faenger ${ }^{1,2}$ \\ ${ }^{1}$ Division of Motor Research, Pathophysiology and Biomechanics, Jena University Hospital, Germany \\ ${ }^{2}$ Department of Experimental Radiology, Jena University Hospital, Germany
}

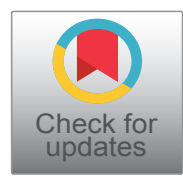

*Corresponding author: Christoph Anders, Clinic for Trauma, Hand and Reconstructive Surgery, Division of Motor Research, Pathophysiology and Biomechanics, Jena University Hospital, Bachstrasse 18, 07743 Jena, Germany, Tel: +493641-934-142, Fax: +49-3641-934-091, E-mail: christoph.anders@med.uni-jena.de

\begin{abstract}
Purpose: Several measures are applied to characterize the functional state of muscles. Among these, the Maximum Voluntary Contraction (MVC) is most frequently used and considered the gold standard. A little understood and used measure is Neuromuscular Efficiency (NME) of muscles. Therefore, the present study was conducted to compare NME indices of several trunk muscles of healthy adults with respect to age and sex.

Methods: Overall 100 healthy Caucasian subjects (equally distributed in young (19-39 years) and elderly (48-71 years) and persons of both sexes, i.e. 25 subjects per age group and sex) were investigated during submaximal and maximal isometric tasks of their trunk muscles in sagittal plane. Five major superficial trunk muscles were evaluated by means of Surface EMG (SEMG). NME and MVC values were extracted. NME was calculated using the inverse slope of the logarithmic transform of the SEMG amplitude to torque relationships of the submaximal tests.

Results: The abdominal muscles showed no sex differences in NME but were always superior in both elderly groups. As could be expected MVC levels during flexion of men were higher but interestingly no age related MVC differences could be detected. However, for the back muscles NME showed no age-related effects and during extension MVC was reduced with age. Males compared with females showed significantly higher NME indices and also higher MVC values. Absolute SEMG amplitudes levels of all investigated trunk muscles were always lower in the elderly subjects.

Conclusions: The NME is an SEMG amplitude independent parameter and may be applied to determine the functional state of (trunk) muscles as a complement to MVC measurements. The observed systematic differences of NME indices between sexes and age groups careful hint towards the NME being related to the functional cross-sectional area of type I fibers.
\end{abstract}

\author{
Keywords \\ Surface Electromyography (SEMG), Amplitude-force rela- \\ tionship, Isometric contraction, Maximum Voluntary Con- \\ traction (MVC)
}

\begin{abstract}
Abbreviations
ANOVA: Analysis of Variance; BMI: Body Mass Index; CSA: Cross Sectional Area; ECG: Electrocardiogram; EMG: Electromyography; LO: Musculus Longissimus; MF: Musculus Multifidus; MVC: Maximum Voluntary Contraction; NME: Neuromuscular Efficiency; OE: Musculus Obliquus Externus; OI: Musculus Obliquus Internus; RA: Musculus Rectus Abdominis; rms: Root Mean Square; SEMG: Surface Electromyography; SFT: Skin Fold Thickness; UBM: Upper Body Mass; UBT: Upper Body Torque
\end{abstract}

\section{Introduction}

Adequate physical fitness reduces the risk of musculoskeletal injuries [1]. Therefore, especially in the light of aging populations improvements in diagnosis of neuromuscular performance are of particular interest. Any biomechanically based diagnosis of neuromuscular function considers power (i.e. explosive strength), force, endurance, co-ordination, and efficiency. In addition, Surface EMG (SEMG) can be used to assess major neuromuscular characteristics like endurance, individual strain level, co-ordination, and efficiency and is therefore increasingly used in research and therapeutic settings [2-5].

In research and assessment of the above mentioned neuromuscular characteristics, baseline data need to be 
established in order to compare subjects or the effect of any kind of intervention such as therapies, training, and nutrition. A simple to take measurement involves determination of Maximum Voluntary Force (MVC). However, an MVC measure can only be an approximation of a subject's force capacity, because it is influenced by motivation $[6,7]$, experience with the required task [8], side dominance [9], tolerance to discomfort and pain [10], age [11], and sex $[12,13]$. Frequently the MVC is taken as the only measure to assess success of interventions. However, exclusive use of MVC ignores the complexity of neuromuscular function.

An additional way to assess the functional state of a muscle or muscle group is the determination of Neuromuscular Efficiency (NME), a straight forward SEMG application $[14,15]$. NME has not been extensively investigated and its importance relative to the other mentioned fitness characteristics is still an open question. But, good neuromuscular efficiency simply stands for the achievement of equal force levels with less expense of energy, or equal muscular strain is accompanied by a higher force output [16]. Consideration of NME dates to the early fifties [1417] and proved to be a predictable measure of the functional state of muscles with respect to sex [18], neurological disorders [19], physical therapy [20], and training $[21,22]$. Initially, NME indices were suggested to be correlated with morphological changes due to hypertrophy in contrast to neural factors $[21,23,24]$. More recently it could be shown that NME indices of the rectus abdominis muscle in healthy subjects were higher for physically active in comparison with inactive women and were also higher in males than in females [18].

Initially, NME was calculated as the inverse slope of the linear regression of the EMG to force relationship at submaximal levels [15]. Later on, this initial approach was refined by using logarithmic transformed EMG data [18] to account for the non-linearity of the EMG to force relationship. Due to the calculation algorithm higher NME indices are associated with less steeper slopes of the SEMG amplitude to torque relationship and therefore represent a better efficiency of the neuromuscular system. The NME can also be determined during MVC tasks but is less accurate and in this case it is the ratio between the respective torque and EMG values during maximum effort.

The calculation of NME indices of trunk muscles in the literature is especially rare. One study identified influences of sex and different physical activity levels on the NME of the rectus abdominis muscle [18], another investigation dealt with the alteration of back muscle NME relative to trunk posture [25], and one further study examined the influence of eccentric exercise [22]. As far as we could find studies that simultaneously determined NME of abdominal and back muscles and related these values with maximum force data are completely lacking.

Therefore, the present study was conducted to compare NME indices of main superficial abdominal as well as back muscles with respect to age and sex, and to relate these data to maximum force values to enhance the interpretation of NME indices. These reference data could be used to improve individual diagnostics of a subject's neuromuscular functional state and could therefore enhance prevention, therapy and rehabilitation.

Since the force capacity of females and males differs largely, we expected to find lower NME indices in women compared with men. Further, older subjects were expected to show lower NME indices compared with young subjects, independent of sex.

\section{Methods}

\section{Subjects}

Overall 100 healthy Caucasian subjects of both sex-

Table 1: Selected anthropometric characteristics and physical activity levels of the study population. Data are given in mean \pm SD.

\begin{tabular}{|c|c|c|c|c|c|c|}
\hline & \multicolumn{2}{|l|}{ Young } & \multirow{2}{*}{$\begin{array}{l}\text { Female vs. } \\
\text { Male }\end{array}$} & \multicolumn{2}{|l|}{ Elderly } & \multirow{2}{*}{$\begin{array}{l}\text { Female vs } \\
\text { Male }\end{array}$} \\
\hline & Female $(n=25)$ & Male $(n=25)$ & & Female $(n=25)$ & Male $(n=25)$ & \\
\hline $\begin{array}{l}\text { age } \\
\text { [years] }\end{array}$ & $26.0 \pm 4.2^{* *}$ & $23.6 \pm 2.2^{* *}$ & n.s. & $59.8 \pm 7.5^{* *}$ & $60.5 \pm 7.3^{* *}$ & n.s. \\
\hline body mass [kg] & $64.8 \pm 4.5$ & $76.8 \pm 9.2^{*}$ & $p<0.001$ & $68.9 \pm 9.7$ & $84.5 \pm 12.7^{*}$ & $p<0.001$ \\
\hline $\begin{array}{l}\text { body height } \\
{[\mathrm{cm}]}\end{array}$ & $167.8 \pm 6.4^{* *}$ & $180.2 \pm 6.2^{* *}$ & $p<0.01$ & $161.9 \pm 6.0^{* *}$ & $175.4 \pm 5.9^{* *}$ & $p<0.01$ \\
\hline $\begin{array}{l}\text { BMI } \\
{\left[\mathrm{kg} / \mathrm{m}^{2}\right]}\end{array}$ & $22.9 \pm 6.4^{* *}$ & $23.6 \pm 2.2^{* *}$ & n.s. & $26.3 \pm 3.4^{\star *}$ & $27.5 \pm 3.9^{* *}$ & n.s. \\
\hline $\begin{array}{l}\text { waist } \\
{[\mathrm{cm}]}\end{array}$ & $74.4 \pm 7.2^{* *}$ & $81.7 \pm 7.4^{* *}$ & $p<0.001$ & $87.0 \pm 8.5^{* *}$ & $97.6 \pm 10.1^{* *}$ & $p<0.001$ \\
\hline $\begin{array}{l}\text { SFT } \\
{[\mathrm{mm}]}\end{array}$ & $54.1 \pm 21.5^{* *}$ & $37.6 \pm 18.9^{* *}$ & $p<0.01$ & $76.8 \pm 22.4^{* *}$ & $64.6 \pm 21.4^{* *}$ & n.s. \\
\hline $\begin{array}{l}\text { UBM } \\
{[\mathrm{kg}]}\end{array}$ & $24.5 \pm 3.8$ & $31.8 \pm 3.4$ & $p<0.001$ & $25.2 \pm 4.5$ & $33.5 \pm 4.9$ & $p<0.001$ \\
\hline $\begin{array}{l}\text { UBT } \\
{[\mathrm{Nm}]}\end{array}$ & $83.5 \pm 17.5$ & $116.1 \pm 17.2$ & $p<0.001$ & $81.7 \pm 19.5$ & $115.7 \pm 24.5$ & $p<0.001$ \\
\hline $\begin{array}{l}\text { PAL } \\
{[1-5]}\end{array}$ & $2.7 \pm 0.8$ & $3.3 \pm 1.0^{* *}$ & $p<0.05$ & $2.9 \pm 0.7$ & $2.6 \pm 0.7^{\star *}$ & n.s. \\
\hline
\end{tabular}

SFT: Skin Fold Thickness (sum of four skin folds, according to [44]); BMI: Body Mass Index; UBM: Upper Body Mass; UBT: Upper Body Torque; PAL: Physical Activity Level. Tests between age groups: " $p<0.05,{ }^{* *} p<0.01$. 


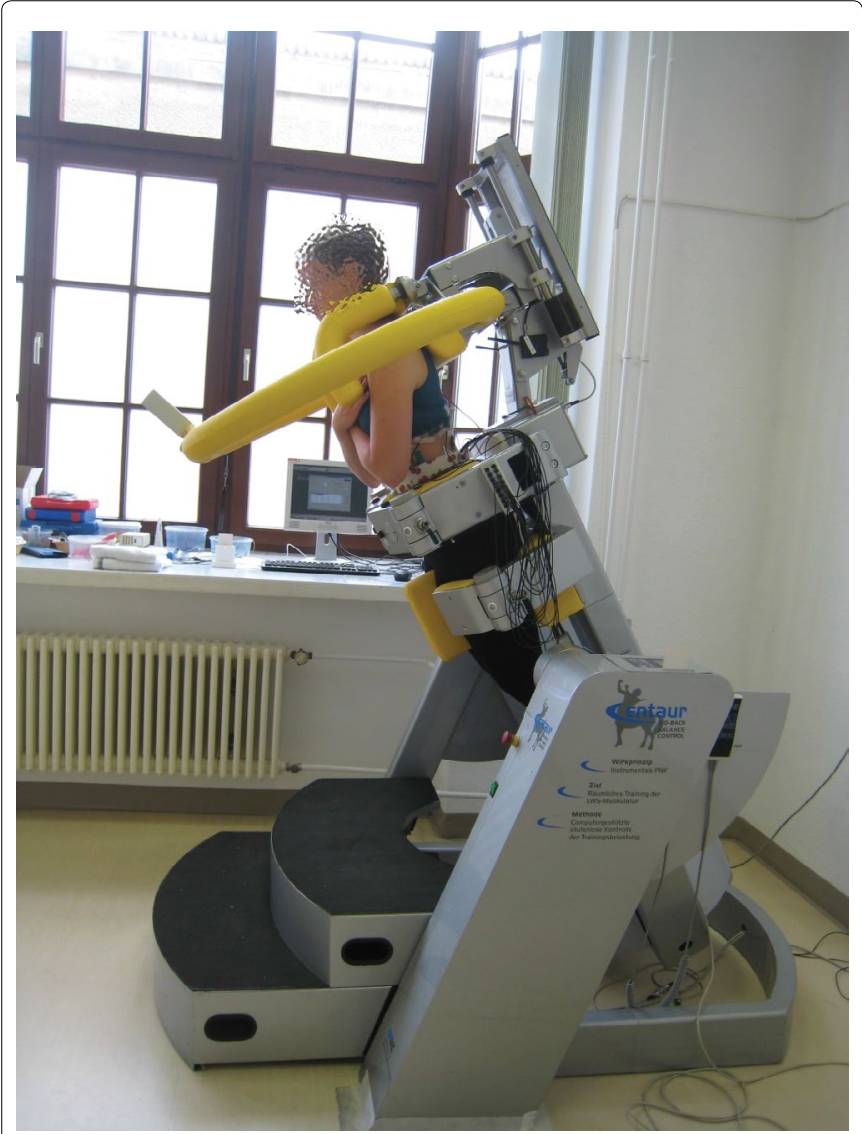

Figure 1: Study setup with a subject positioned in the device, performing a $30^{\circ}$ forward tilt.

es, equally distributed in young (19-39 years, mean: $27.3 \pm 5.0$ years) and elderly subjects ( $48-71$ years, mean $60.1 \pm 7.3$ years) were included in the study. Detailed information about the study purpose and procedure was provided. Informed consent was obtained from all individual participants included in the study. The study protocol was approved by the ethics committee of the Jena University Hospital (2643-08/09). All participants were clinically examined and asked about their medical history. Exclusion criteria were actual back or radiating pain, any surgery of the spinal column, and also any $\mathrm{C}$-section. Selected anthropometric characteristics are given in Table 1. The level of physical activity on a 1 to 5 self-rating scale was also obtained from all subjects (see Table 1 ). In this scale 1 stands for "no physical activity at all" and 5 is rated as "extremely high level of physical activity" (daily high intensity sports or hard physical work for at least one hour).

\section{Testing overview}

All tests were conducted in a computerized test and training device for whole body tilt (CENTAUR, BfMC, Germany). In this device the subject's lower body is fixed at hip and thigh, whereas the upper body remains free (Figure 1). For the submaximal test conditions portions of the Upper Body Mass (UBM) were applied by tilting the subject from vertical position at defined tilt angels. During these tasks the subject had to remain in upright body posture and therefore simply to compensate the gravitational forces acting on the trunk (Figure 1). Correct adherence to upright body position was enabled by a harness, positioned over the subject's shoulder that was equipped with strain gauges. A biofeedback monitor in front of the subject contained a crosshair and a point that was deviated from the center of the crosshair if any force was applied to the harness, i.e. the person failed to maintain the desired upright posture. Exact adherence to the mentioned conditions was controlled by the investigator and assisted by correction suggestions if necessary. The Maximum Voluntary Contraction (MVC) tests were also performed in the device with subjects standing upright.

The applied submaximal test conditions consisted of isometric applications of $9 \%, 17 \%, 34 \%, 50 \%, 71 \%, 87 \%$, and $100 \%$ of the Upper Body Mass (UBM) and were realized by tilting the subjects by $5^{\circ}, 10^{\circ}, 20^{\circ}, 30^{\circ}, 45^{\circ}, 60^{\circ}$, and $90^{\circ}$ from vertical position in sagittal plane, i.e. in forward and backward directions. These tilt angles were chosen as a compromise between the possibility of exactly adjustable tilt angles and desired equally spaced torque moment differences. All 14 tasks were applied in an individually randomized order to prevent possible order related effects. While the device moved (i.e. tilting to reach the desired position) subjects leaned relaxed on the harness and were asked to move away from the harness and adopt upright body position if the target angle was reached. Every submaximal task was executed for approximately ten seconds to ensure isometric conditions. The submaximal tests incidentally provided a necessary warmup for obtaining accurate force levels during the MVC test. After completing the submaximal tests, subjects then immediately performed at least one halfhearted practice force test that was followed by three single MVC trials of which the best was used for analysis [26]. For this subject were asked to apply their maximum trunk extension and flexion forces against the harness for approximately three seconds during standing with one-minute interval between each test. Subjects were informed that there would be three MVC trials [26] and strong verbal encouragement was provided [7]. MVC execution was observed religiously and corrected to ensure proper execution. The MVC force data were already registered during the tests. If conspicuous data were observed, i.e. the variability between the tests exceeded more than $10 \%$ the MVC test was repeated $[27,28]$.

During all test situations the subjects remained with arms crossed over chest to avoid effects due to varying arm positions.

The UBM of all subjects was determined while the device was tilted forward to $90^{\circ}$ and participants lay relaxed on the harness. At this position the strain gauge values corresponded to the subjects' UBM. To ensure plausible values full relaxation of the back muscles was controlled via Surface EMG (SEMG). Hints were provid- 
Table 2: SEMG Electrode positions.

\section{Muscle}

M. rectus abdominis (RA)

M. obliquus internus $(\mathrm{OI})$

M. obliquus externus (OE)

M. multifidus (MF)

M. longissimus (LO)

ECG

\section{Localization/orientation}

Caudal electrode at navel height, $4 \mathrm{~cm}$ from center/vertical

Medial inguinal ligament, at anterior superior iliac spine height/horizontal

Cranial electrode directly below lowest point of the costal arch/along line to contralateral pubic tubercle

Caudal electrode at $\mathrm{L} 5$ height $/ 1 \mathrm{~cm}$ medial and parallel to line between posterior superior iliac spine line and L1

Caudal electrode at L1 height, over palpable bulge of muscle (approx. 2 fingers lateral from midline)/vertical

Above heart/along heart axis ed if remaining activity could be detected. This procedure was repeated two times and the highest value was further used in the analysis. Additionally, the distance between the iliac crest and the projection of the scapular spine to the spine was determined along the spine and further used to calculate the individual upper body torque (UBT, see Table 1) and MVC torque values.

\section{Measurements and data analysis}

Bipolar SEMG was taken from five superficial trunk muscles, simultaneously from both body sides: M. Rectus Abdominis (RA), M. Obliquus Internus Abdominis (OI), M. Obliquus Externus Abdominis (OE), M. Multifidus Lumbalis (MF), and M. erector spinae, Pars Longissimus (LO). All electrode positions are detailed in Table 2 and were chosen in accordance with the accepted international recommendations and $\mathrm{Ng}$ [29-31]. Additionally, the cardiac activity was detected by the application of a further electrode pair along the heart axis for subsequent elimination of the inevitable ECG- contamination of the SEMG signals. Prior to SEMG electrode application the respective regions were marked by an experienced examiner, shaved if necessary, and cleaned with abrasive paste (Epicont, GE, Germany). The used electrodes had a circular uptake area of $1.6 \mathrm{~cm}$ diameter (H93SG, Covidien, Germany) and an inter-electrode distance of $2.5 \mathrm{~cm}$. The SEMG signals were amplified (gain: 1000, input impedance: $1200 \mathrm{G} \Omega$, noise level: $<1 \mu \mathrm{V}$, CMRR > $120 \mathrm{~dB}, 10-700 \mathrm{~Hz}, 1^{\text {st }}$ order RC filter, Biovision, Germany), analog to digital converted (2000/s, Tower of Measurement, 24 bit resolution at $\pm 5 \mathrm{~V}$ : $0.6 \mathrm{nV} / \mathrm{bit}$, anti-aliasing filter at $1000 \mathrm{~Hz}$, DeMeTec, Germany), collected (ATISArec, GJB, Germany) and stored on hard drive for offline processing. The MVC force data were determined by simultaneously capturing the strain gauge values together with the SEMG data. To determine the MVC force levels steady sections of the force graph correlating to the three MVC trials were marked. For each of these sections the mean was calculated, and the highest value was used as the respective MVC force level.

Raw data were band-pass filtered between $10 \mathrm{~Hz}$ and $300 \mathrm{~Hz}$. Possible interferences from the power supply were eliminated by a $50 \mathrm{~Hz}$ notch filter. To eliminate the inevitable ECG contamination of the SEMG signals only steady-state sections of $400 \mathrm{~ms}$, starting at an interval of 100 ms subsequent to all determined R-waves, were considered for analysis. The average value of all respective segments was used to calculate the representative root mean square ( $\mathrm{rms}$ ) values per trial and muscle. The NME of the abdominal muscles was determined using data from all backward tilt positions (i.e. isomeric trunk flexion tasks) and for the NME of back muscles all the forward tilt data were combined (i.e. isomeric trunk extension tasks).

\section{Determination of the NME}

NME can be calculated from either MVC tasks or a series of submaximal tasks. To determine the NME during MVC tasks the developed torque or force is related to the respective rms value $[32,33]$. In our investigation we calculated NME by using a series of submaximal tasks, similar to the approach of David and colleagues [18]. This at first required the identification of the best fit for the respective rms to torque relationships. A second order polynomial function resulted in the best fit (coefficient of determination $\left(r^{2}\right) 0.971$ to 0.991; mean: $0.984, S D \pm 0.006$ ) for all muscles, in both sexes and age groups. The rms values were then linearized by application of the natural logarithm. From these logarithmic transformed rms data individual linear regression slopes were fitted. The mean NME for every muscle, sex, and age group was determined by averaging the inverse values of the respective individual linear slopes.

\section{Statistical analysis}

At first, a repeated measures ANOVA was performed to detect if any influence of body side on NME had to be considered and it was deemed there is no side influence. Therefore, pooled values from both sides were used for all subsequent analyses.

Prior to the detailed statistical analyses a univariate ANOVA was performed to identify general influences of muscle, sex, and age on NME. Specific tests for group differences of NME indices were performed by the application of Student's t-tests for independent samples. The significance level was set to $p<0.05$. All analyses were conducted with IBM SPSS Statistics 21.0 (IBM, NY, USA).

\section{Results}

The univariate ANOVA of the NME indices showed significant influences of age $(F=6.849, p=0.009)$, $\operatorname{sex}(F=89.392, p<0.001)$, and muscle $(F=215.395$, 
Table 3: NME indices of the investigated trunk muscles.

\begin{tabular}{|c|c|c|c|c|c|c|}
\hline & \multicolumn{2}{|c|}{ Young } & \multirow{2}{*}{$\begin{array}{l}\text { Female vs. } \\
\text { Male }\end{array}$} & \multicolumn{2}{|c|}{ Elderly } & \multirow{2}{*}{$\begin{array}{c}\text { Female vs. } \\
\text { Male }\end{array}$} \\
\hline & Female & Male & & Female & Male & \\
\hline RA & $20.9 \pm 6.9$ & $21.4 \pm 3.7^{\star *}$ & n.s. & $22.6 \pm 6.7$ & $26.0 \pm 7.5^{\star *}$ & n.s. \\
\hline $\mathrm{Ol}$ & $30.0 \pm 10.0^{*}$ & $31.6 \pm 9.0^{*}$ & n.s. & $40.2 \pm 17.7^{*}$ & $40.9 \pm 15.9^{*}$ & n.s. \\
\hline OE & $32.4 \pm 10.3$ & $32.9 \pm 5.6$ & n.s. & $33.5 \pm 9.1$ & $37.7 \pm 10.6$ & n.s. \\
\hline MF & $50.4 \pm 12.4$ & $76.6 \pm 17.8$ & $p<0.001$ & $51.9 \pm 17.8$ & $81.9 \pm 18.9$ & $p<0.001$ \\
\hline LO & $64.3 \pm 15.4$ & $94.9 \pm 28.7$ & $p<0.001$ & $59.6 \pm 21.4$ & $99.1 \pm 35.7$ & $p<0.001$ \\
\hline
\end{tabular}

Data are presented as mean \pm SD. Tests between age groups: ${ }^{*} p<0.05,{ }^{* *} p<0.01$.

SEMG amplitude to torque relationship: polynomial trends logarithmic SEMG amplitude to torque relationship: linear trends
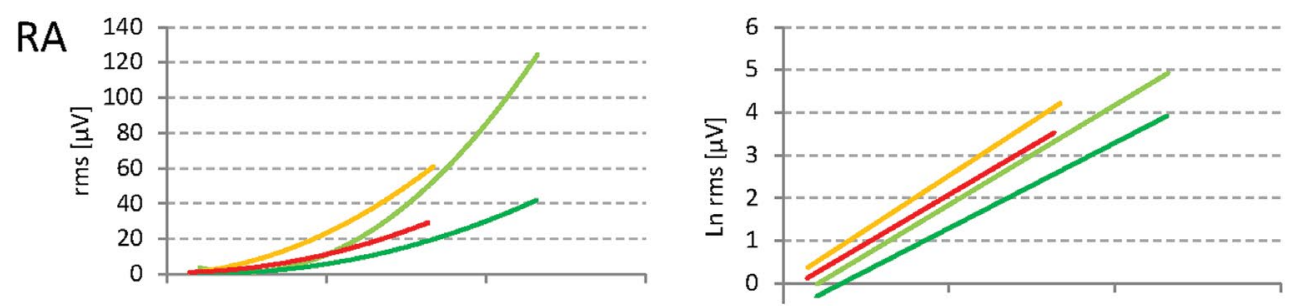

$\mathrm{Ol}$
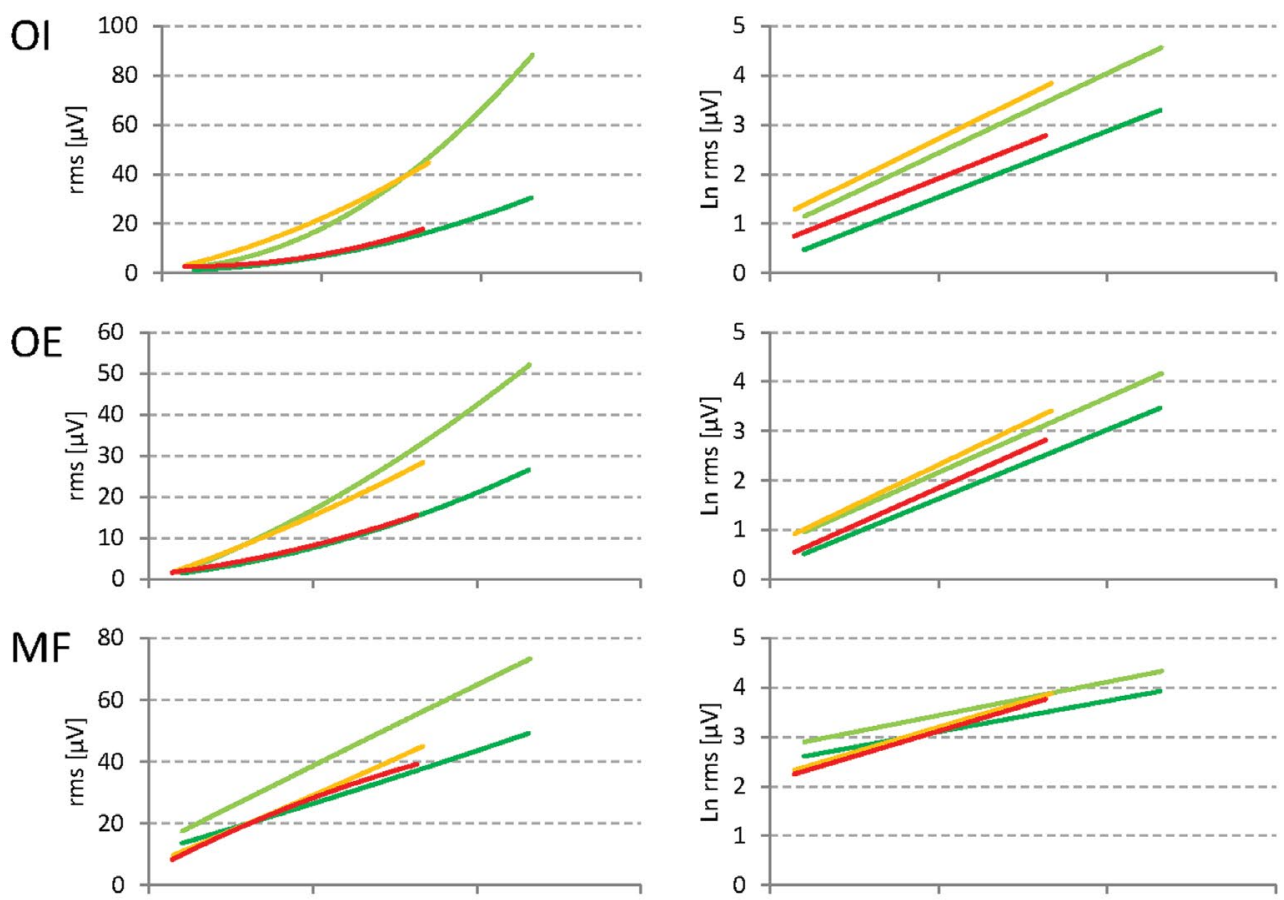

LO
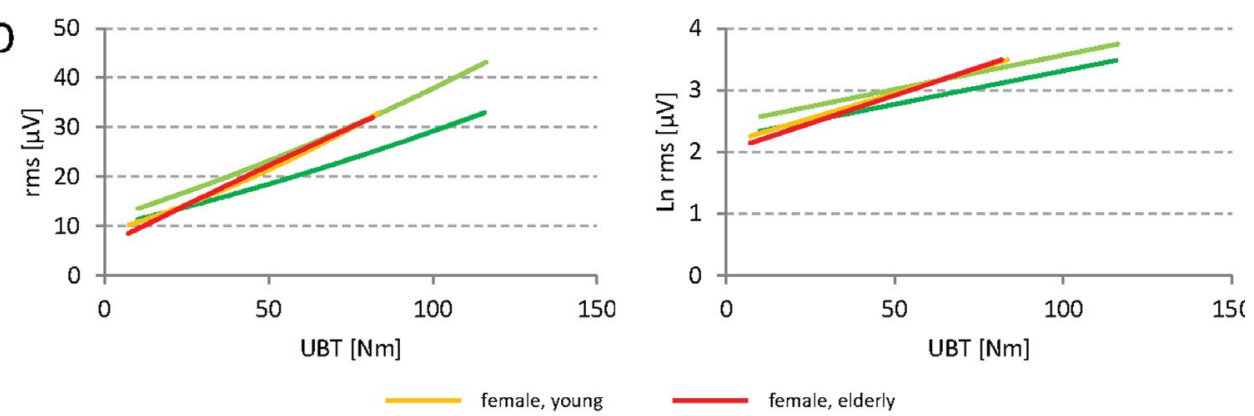

female, young

male, young

male, elderly

Figure 2: Trend curves for the SEMG to torque relationships of portions of the upper body mass expressed as Upper Body Torque (UBT) values. Left: polynomial trend curves of the respective mean SEMG amplitude to torque relationship, Right: linear trend curves of the logarithmic transforms of the mean SEMG amplitude to torque relationship.

$p<0.001)$, with a significant but ordinal interaction between sex and muscle $(F=25.811, p<0.001)$. Therefore, muscles were further analyzed individually and separately for sex and age.
The NME indices of the abdominal muscles differed with age, always being higher in the elderly subjects. This could significantly be shown in males for the RA and Ol and in females for the OI (Table 3). In contrast, female 
male

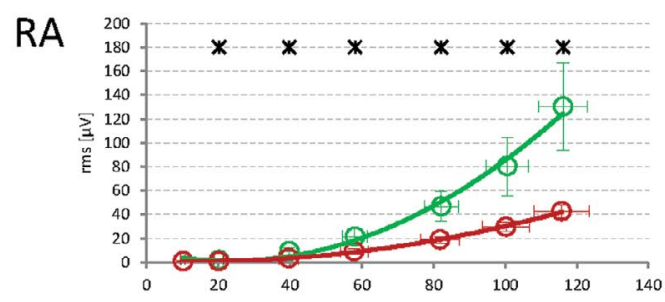

Ol

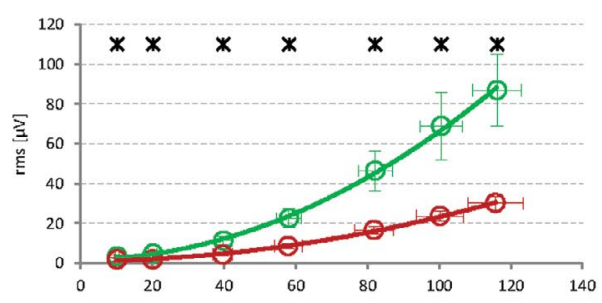

OE
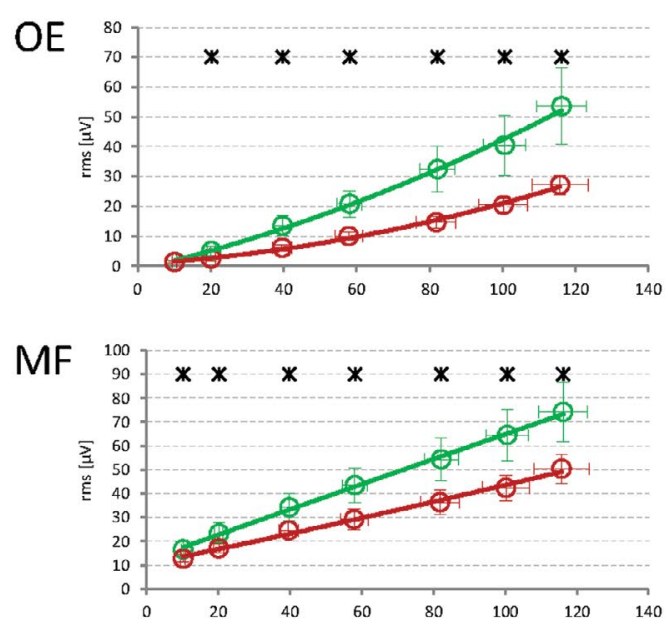

LO

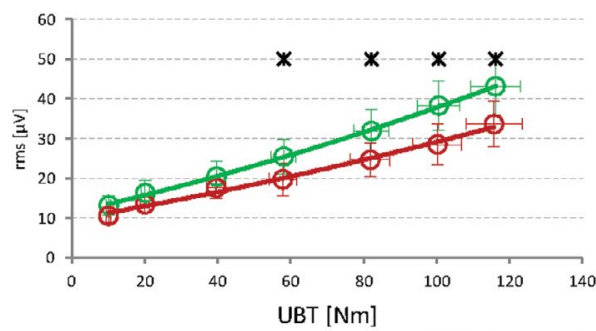

female
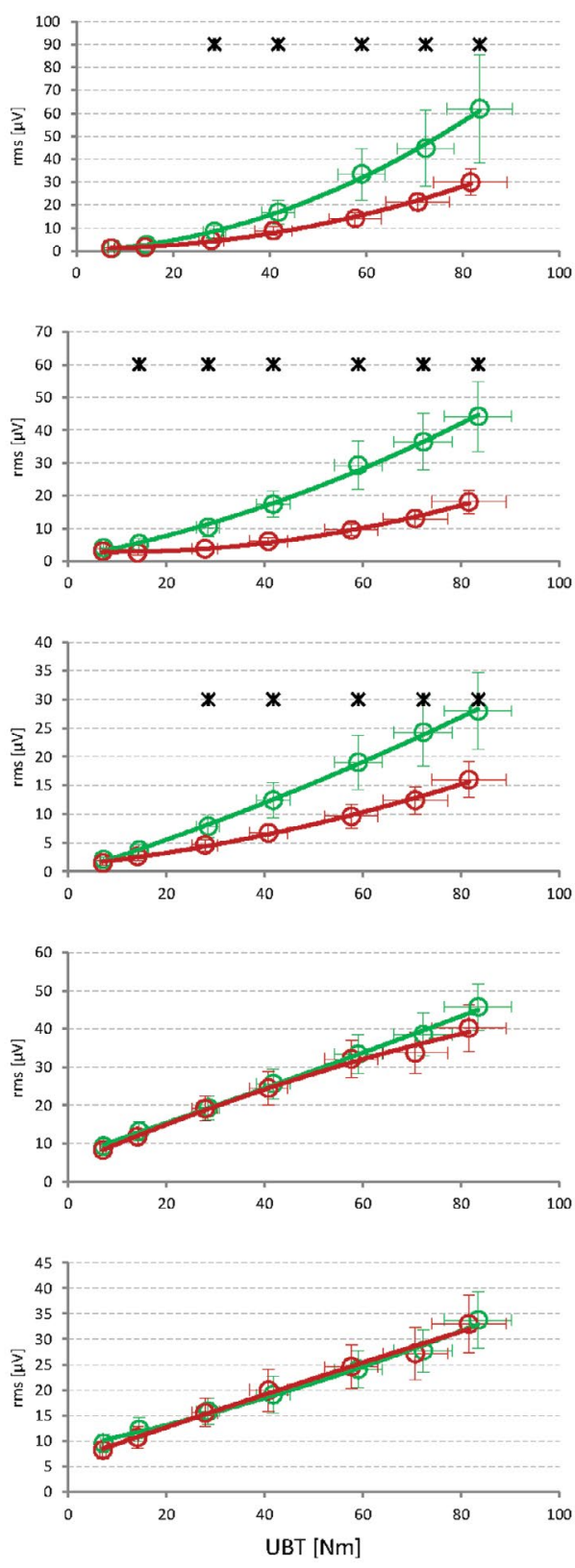

Figure 3: SEMG amplitude to torque relationships of the investigated male (left) and female (right) subjects. The statistics were performed under the assumption of equal UBT values. All data are given as mean \pm SD.

Table 4: MVC torque levels.

\begin{tabular}{|l|c|c|c|c|c|c|}
\hline & \multicolumn{2}{|c|}{ Young } & \multicolumn{2}{c|}{$\begin{array}{c}\text { Female vs. } \\
\text { Male }\end{array}$} & \multicolumn{2}{c|}{$\begin{array}{c}\text { Elderly } \\
\text { Female vs. } \\
\text { Male }\end{array}$} \\
\hline flexion & Female & Male & & Male & Male & $p<0.001$ \\
\hline extension & $124.7 \pm 37.0$ & $220.6 \pm 49.8$ & $\mathrm{p}<0.001$ & $126.3 \pm 31.2$ & $215.1 \pm 44.4$ & $\mathrm{p}<0.2$ \\
\hline
\end{tabular}

Torque data are presented in $\mathrm{Nm}$. All data are given as mean $\pm \mathrm{SD}$.

Tests between age groups: ${ }^{*} p<0.05,{ }^{* *} p<0.01$.

subjects always showed significantly lower NME indices for the back muscles independent of age.

The relationship between the SEMG amplitudes together with their logarithmic transforms versus the respective torque levels are detailed in Figure 2. In males SEMG amplitudes at almost all submaximal torque levels differed between the two age groups, being always higher in the young group (Figure 3 ). However, in females significant SEMG amplitude differences could only be observed in the abdominal muscles, again with higher levels in the young group (Figure 3 ).

For both flexion and extension muscle groups the MVC torque levels were always significantly higher in male subjects. For the maximum extension task young women showed significantly higher torque levels in comparison to the elderly female group (Table 4). 


\section{Discussion}

The actual investigation determined NME indices of trunk muscles at submaximal torque levels that were induced by graded tilts in sagittal plane. For the abdominal muscles NME indices were always higher in the elderly subjects, this was especially pronounced in the male group. Interestingly, for the back muscles no systematic age-related influences of the NME could be proven, but women in comparison with men showed significantly lower NME indices. As expected, men in comparison with women always showed higher MVC levels. Interestingly, the MVC levels during flexion (abdominal muscles) were almost identical between both age groups, but for the maximum extension (back muscles) the young groups both performed better - significantly so for the female subjects.

\section{Validity of data}

The NME data of the present study are in good agreement with the results of David, et al. [18] and this substantiates a trustworthy basis for the present investigation. They determined NME indices of the RA and MVC flexion torque levels in female gymnasts, male runners, and normally active controls of both sexes. The young males' NME indices of the RA in the present study are positioned just midway between the NME indices of the male controls and the runners of the David study. The NME indices of our young female group correspond more to the group of gymnasts and not their normal female controls. As to the MVC torque data our female young group's data perfectly match with the Davis young normal controls data, but our young males performed more like the male runners. Because both MVC and NME data of our young males were better than in the David investigation it might be that our young males were somehow fitter. To further support the correspondence of these two studies, the BMI of our young groups of both sexes fit nicely with those of the respective sex groups [18].

\section{Is the NME a redundant parameter?}

If NME is redundant with either MVC and SEMG amplitudes then one would expect concordant differences between age groups and between sexes, but this is not the case: NME, independent of sex, increased with age for all tested muscles for both flexion and extension, while MVC during extension together with all SEMG amplitude levels decreased with age and remained virtually unchanged during flexion. By looking at sex differences we found the NME to be higher in males for the back muscles only. As expected MVC levels, again together with the SEMG amplitude levels, were always higher in males in both flexion and extension exercises.

\section{Morphofunctional background}

With increasing age, due to age related involution, there is a loss of alpha and gamma motor neurons
$[34,35]$ together with a numerical atrophy that is particularly pronounced in type II fibers [36]. Data from healthy young subjects of both sexes are available detailing fiber type and distribution of thoracic and lumbar back muscles [37]. Mannion and colleagues showed that the cross-sectional area of all muscle types was considerably larger in men [37]. Further, the ratio of type I fibers is much higher in back muscles as compared with the abdominals $[38,39]$.

Combining these morphological facts helps to hypothetically explain the found differences in NME indices, since they are in concordance with the expected Cross Sectional Area (CSA) of type I fibers according to age, sex, and specific muscle (i.e. localization).

Beside the involution related arguments, the elderly subjects showed an elevated BMI. This was independent of sex. By definition the calculation of the NME is independent from amplitude level. At this point it cannot be excluded that the reduced amplitudes in the elderly may have yet influenced the results, most probably due to irregular dampening conditions for low and high muscular strain levels. This argument also remains hypothetical, since to the authors' knowledge no systematic investigations exist in this regard.

\section{Limitations}

Since we did not measure the fiber-type distribution in our subjects the given hypothetical interpretation of NME still contains a missing link that has to be closed by future investigations.

The present study was conducted using the CENTAUR that applies graded forces on the trunk by tilting the subjects in sagittal plane. This offers the advantage of highly reliable testing, but still is an artificial situation.

A second order polynomial function was the best fit of the amplitude to torque relationship of all investigated trunk muscles. However, the non-transformed SEMG values the back muscles showed a very low quadratic coefficient (mean values MF: -0.00023 , LO: 0.00046 ) that would allow the application of a linear regression with high accuracy [40], but one would not have been able to directly compare NME data between back and abdominal muscles. Therefore, by generally using the logarithmic transformed data any unaware error due to misleadingly applied linear calculations for the non-transformed values of the abdominal muscles can be avoided.

\section{Conclusions}

Summarizing all mentioned aspects NME indices might be a correlate of the functional cross sectional area of type I fibers: The number of type I fibers is always higher in the back muscles and the CSA of all fiber types is larger in men. Further, with increasing age primarily the number of type II fibers is reduced. This affects the abdominal muscles first since they contain a larger proportion of type II fibers. 
The NME seems to be a force independent but morpho-functionally linked characteristic of muscle function that is independent from SEMG amplitude level and maximum force (MVC). It may therefore be applied as a physiologically derivable indicator of the type I to type II ratio, but this is currently speculative. Based on the known age-related change in fiber type, it may be reasonable to infer that the elderly showed higher NME indices of their abdominal muscles and men showed larger NME indices of back muscles than women.

SEMG amplitudes are influenced by many factors. Because of this it is recommended to normalize SEMG amplitudes. To account for interindividual differences in this study we measured the BMI and the SFT. Neither one of these was able to explain all the observed differences. This is in agreement to previous theoretical $[41,42]$ and practical [43] results: Accounting for subcutaneous fat failed to sufficiently explain interindividual amplitude variance of SEMG amplitudes $[43,44]$.

\section{Practical Applications}

The NME of trunk muscles shows differences according to sex, age, and specific muscle. The calculated NME indices are robust against both systematic SEMG amplitude differences and MVC levels. The NME therefore offers a promising complementary, physiologically based measure to assess the functional state of trunk muscles that may indirectly hint at fiber type distribution, although at this point this is mostly speculative. The NME could then be used to specifically guide and monitor rehabilitation and training. In as far as how well a certain protocol is supporting the increase of the one or the other type of muscle fiber (type I for increased strength and type II for increased stability) the NME would then change or not. Anyhow, this hypothesis needs to be proven by specific training interventions.

\section{Acknowledgements}

The authors wish to thank all the subjects for their time and effort. Measurements were carried out at the kindly provided laboratory of the Center for Interdisciplinary Prevention of Diseases related to Professional Activities (KIP) funded by the Friedrich-Schiller-University Jena and the German Social Accident Insurance Institution for the foodstuffs and catering industry. The authors also gratefully acknowledge the linguistic and intellectual support by Ms. Marcie Matthews of polished words.

\section{Funding}

The study was supported by the Central Innovation Program of the German Federal Ministry of Economics and Technology, Grant KF2150501WD8. The study sponsors had no involvement in the study design, the collection, analysis and interpretation of data, or in the writing of the manuscript. Hereby all authors decline any financial and personal relationships with other people or organizations.

\section{References}

1. Troup JD (1984) Causes, prediction and prevention of back pain at work. Scand J Work Environ Health 10: 419-428.

2. De Luca CJ (1993) Use of the Surface Emg Signal for Performance Evaluation of Back Muscles. Muscle Nerve 16: 210-216.

3. Farina D, Merletti R, Enoka RM (2004) The extraction of neural strategies from the surface EMG. J Appl Physiol (1985) 96: 1486-1495.

4. Kleissen RF, Hermens HJ, den Exter T, de Kreek JA, Zilvold G (1989) Simultaneous measurement of surface EMG and movements for clinical use. Medical \& Biological Engineering \& Computing 27: 291-297.

5. Merlo A, Farina D, Merletti R (2003) A fast and reliable technique for muscle activity detection from surface EMG signals. IEEE Trans Biomed Eng 50: 316-323.

6. Andreacci JL, LeMura LM, Cohen SL, Urbansky EA, Chelland SA, et al. (2002) The effects of frequency of encouragement on performance during maximal exercise testing. J Sports Sci 20: 345-352.

7. McNair PJ, Depledge J, Brettkelly M, Stanley SN (1996) Verbal encouragement: effects on maximum effort voluntary muscle action. Br J Sports Med 30: 243-245.

8. Jones TW, Howatson G, Russell M, French DN (2013) Performance and Neuromuscular Adaptations Following Differing Ratios of Concurrent Strength and Endurance Training. J Strength Cond Res 27: 3342-3351.

9. Noguchi T, Demura S, Takahashi K, Demura G, Mori $Y$ (2014) Differences in Muscle Power between the Dominant and Nondominant Upper Limbs of Baseball Players. J Strength Cond Res 28: 82-86.

10. Chiou SY, Shih YF, Chou LW, McGregor AH, Strutton PH (2014) Impaired neural drive in patients with low back pain. Eur J Pain 18: 794-802.

11. Doherty TJ, Vandervoort AA, Taylor AW, Brown WF (1993) Effects of motor unit losses on strength in older men and women. J Appl Physiol (1985) 74: 868-874.

12. Behm DG, Sale DG (1994) Voluntary and evoked muscle contractile characteristics in active men and women. Can J Appl Physiol 19: 253-265.

13. Kaplanis PA, Pattichis CS, Hadjileontiadis LJ, Roberts VC (2009) Surface EMG analysis on normal subjects based on isometric voluntary contraction. J Electromyogr Kinesiol 19: 157-171.

14. Lippold OCJ (1952) The Relation between Integrated Action Potentials in a Human Muscle and Its Isometric Tension. J Physiol 117: 492-499.

15. DeVries HA (1968) "Efficiency of electrical activity" as a physiological measure of the functional state of muscle tissue. Am J Phys Med 47: 10-22.

16. Moritani T, deVries HA (1978) Reexamination of the relationship between the surface integrated electromyogram (iemg) and force of isometric contraction. Am J Phys Med 57: 263-277.

17. Fischer A, Merhautova J (1961) Electromyographic manifestations of individual stages of adapted sports technique. Health and Fitness in the Modern World. 61. Chicago: The Athletic Institute, Library of the Congress.

18. David P, Mora I, Perot C (2008) Neuromuscular Efficiency of the Rectus Abdominis Differs with Gender and Sport 
Practice. Journal of Strength and Conditioning Research 22: 1855-1861.

19. Lenman Ja (1959) A clinical and experimental study of the effects of exercise on motor weakness in neurological disease. J Neurol Neurosurg Psychiatry 22: 182-194.

20. Bradbury-Squires DJ, Noftall JC, Sullivan KM, Behm DG, Power KE, et al. (2015) Roller-Massager Application to the Quadriceps and Knee-Joint Range of Motion and Neuromuscular Efficiency During a Lunge. J Athl Train 50: 133140.

21. Moritani T, deVries HA (1979) Neural factors versus hypertrophy in the time course of muscle strength gain. Am J Phys Med 58: 115-130.

22. Hermann KM, Barnes WS (2001) Effects of eccentric exercise on trunk extensor torque and lumbar paraspinal EMG. Med Sci Sports Exerc 33: 971-977.

23. Laycoe RR, Marteniuk RG (1971) Learning and tension as factors in static strength gains produced by static and eccentric training. Res Q 42: 299-306.

24. Ikai M, Steinhaus AH (1961) Some factors modifying the expression of human strength. J Appl Physiol 16: 157-163.

25. Roy AL, Keller TS, Colloca CJ (2003) Posture-dependent trunk extensor EMG activity during maximum isometrics exertions in normal male and female subjects. J Electromyogr Kinesiol 13: 469-476.

26. Kurz E, Anders C, Walther M, Schenk P, Scholle HC (2014) Force capacity of back extensor muscles in healthy males: effects of age and recovery time. J Appl Biomech 30: 713721.

27. Luoto S, Hupli M, Alaranta H, Hurri H (1996) Isokinetic performance capacity of trunk muscles. Part II: Coefficient of variation in isokinetic measurement in maximal effort and in submaximal effort. Scandinavian Journal of Rehabilitation Medicine 28: 207-210.

28. Birmingham TB, Kramer JF, Speechley M, Chesworth BM, MacDermid J (1998) Measurement variability and sincerity of effort: clinical utility of isokinetic strength coefficient of variation scores. Ergonomics 41: 853-863.

29. Hermens HJ, Freriks B, Disselhorst-Klug C, Rau G (2000) Development of recommendations for SEMG sensors and sensor placement procedures. J Electromyogr Kinesiol 10: 361-374.

30. Hermens HJ, Freriks B, Merletti R, Stegeman DF, Blok J, et al. (1999) European Recommendations for Surface ElectroMyoGraphy, results of the SENIAM project. Enschede: Roessingh Research and Development.

31. Ng JK, Kippers V, Richardson CA (1998) Muscle fibre orientation of abdominal muscles and suggested surface EMG electrode positions. Electromyogr Clin Neurophysiol 38: 51 58.

32. Arabadzhiev TI, Dimitrov VG, Dimitrova NA, Dimitrov GV (2010) Interpretation of EMG integral or RMS and estimates of "neuromuscular efficiency" can be misleading in fatiguing contraction. J Electromyogr Kinesiol 20: 223-232.

33. Magalhaes I, Bottaro M, Mezzarane RA, Neto FR, Rodrigues $\mathrm{BA}$, et al. (2016) Kinesiotaping enhances the rate of force development but not the neuromuscular efficiency of physically active young men. J Electromyogr Kinesiol 28: 123-129.

34. Terao S, Sobue G, Hashizume Y, Li M, Inagaki T, et al. (1996) Age-related changes in human spinal ventral horn cells with special reference to the loss of small neurons in the intermediate zone: A quantitative analysis. Acta Neuropathol 92: 109-114.

35. Kawamura Y, Obrien P, Okazaki H, Dyck PJ (1977) Lumbar Motoneurons of Man .2. Number and Diameter Distribution of Large-Diameter and Intermediate-Diameter Cytons in Motoneuron Columns of Spinal-Cord of Man. J Neuropath Exp Neur 36: 861-870.

36. Deschenes MR (2004) Effects of aging on muscle fibre type and size. Sports Med 34: 809-824.

37. Mannion AF, Dumas GA, Cooper RG, Espinosa FJ, Faris MW, et al. (1997) Muscle fiber size and type distribution in thoracic and lumbar regions of erector spinae in healthy subjects without low back pain: normal values and sex differences. J Anat 190: 505-513.

38. Häggmark T, Thorstensson A (1979) Fibre types in human abdominal muscles. Acta Physiol Scand 107: 319-325.

39. Thorstensson A, Carlson H (1987) Fibre types in human lumbar back muscles. Acta Physiol Scand 131: 195-202.

40. Anders C, Brose G, Hofmann GO, Scholle HC (2008) Evaluation of the EMG-force relationship of trunk muscles during whole body tilt. J Biomech 41: 333-339.

41. Stegeman DF, Blok JH, Hermens HJ, Roeleveld K (2000) Surface EMG models: properties and applications. J Electromyogr Kinesiol 10: 313-326.

42. De Luca CJ (1979) Physiology and mathematics of myoelectric signals. IEEE Trans Biomed Eng 26: 313-325.

43. Nordander C, Willner J, Hansson GA, Larsson B, Unge J, et al. (2003) Influence of the subcutaneous fat layer, as measured by ultrasound, skinfold calipers and BMI, on the EMG amplitude. Eur J Appl Physiol 89: 514-519.

44. Durnin JV, Womersley J (1974) Body fat assessed from total body density and its estimation from skinfold thickness: measurements on 481 men and women aged from 16 to 72 years. Br J Nutr 32: 77-97. 